\title{
Design of Simulated Software for Analysis and Studies of Computer organization
}

\author{
${ }^{*}$ P. Surya Chandra \\ Ramesh P \\ GIT, GITAM University, \\ Visakhapatnam
}

\author{
K.V. Ramana Rao \\ Pradeep U.K \\ Pydah College Of Engineering \\ and \\ Technology, Visakhapatnam.
}

\author{
L. N. Chaitanya \\ Sanketika Vidyaparishad engg \\ College
}

\begin{abstract}
In some cases it is very difficult to understand real time application by the student. It is also tedious job to construct a circuit. In such situations simulation software plays vital role in understanding output of digital circuits by virtually constructing the circuit. In the present paper new simulated software is presented to understand and analyse the computer organization and its applications. The present simulation software is also use full for researchers to understand the behaviour and further need of modifications in their design.
\end{abstract}

\section{Keywords}

Simulation, testing, Software, microprocessor, Computer organization

\section{INTRODUCTION}

In current software process, Software Design Simulations are anticipated to be used to help,the design real time applications virtually more systematically. Systematic development processes is an important characteristic of any advanced engineering discipline. This paper describes the work that is aimed at Software Simulation Design processes more systematic and thus more effective in guiding designers, students and researchers. This work begins with the assumption that the large diversity of Software Simulation Design Methods provides with such well-defined and systematic design processes. This paper concentrates on the Object Oriented Design Programing Method to elaborate the virtual world of computer organization. As design is perhaps the most crucial task in software development, it seems particularly important that software design processes be clearly defined in such a way as to be more systematic and creative. The work builds upon the basic ideas of object oriented programming, to explain basic building block of computer operations. The Booch Object Oriented Design process is supportable for single users working on a single design project [7]. In the present design elaborated versions are used, so that multi users can work at a time on different designs. In the present work graphics tools are used to explain the signal concepts like transmitting and receiving of data, requests, acknowledgements, interrupts and much more. New header file is created to define reusable repeated functions. These are very much useful while developing the code to reduce the executable time and program size. The component design is highly used in the present work for ease of implementation and efficient process. A component is a functional element of a program that combines processing logic, the internal data structures required to implement the processing logic, and an interface that enables the component to be invoked and data to be passed to it. Also good interface techniques are used for well-established work flow. Interfacing in design process will link from one page to other pages or one application to other applications. Finally the design interface must be user friendly. The links must be meaning full and must invoke related page.

\section{MOTIVATION}

In the present days the graduate levels are not mapping the present industrial needs. The prior industrial oriented training work is required to full fill their engineering knowledge and to meet industrial demands. But it is very difficult to gain industrial oriented experience prior to become an employ in any industry. Simulation soft-wares can then be play vital role in gaining the industrial oriented experience.

In developing countries, most of the educational institutions following blended learning [8]. Sustainable E-learning plays an important role in all cultures of blended learning. E-learning substantially improves and expands the learning opportunities for students [2]. The moderncomputer information technologies, which are widely used both at educational programs for conducting of effective lecture, conducted scientific researches, and forming of practical and laboratory works with the students of technical and computer-based specialdisciplines[3].

The researchers really face difficult in studying and identifying the faults in the circuits. And it is highly essential to study the behaviour of the signals present in the system. Bus it is very difficult to construct or modify the hardware every time when their out does not meet the requirement. Simulations are best solution for this to do their research in best way. But ultimately the real time applications only give the final results.

\section{DESIGN AND CODING}

The design is created such that it is user friendly and easy to understand. Multiple runs are effectively done by easy interface with user. A Small window (Message Box) module is like as follows,

unsigned window_size;

void * window_buffer;

window_size=imagesize $(160,100,430,250)$;

window_buffer=malloc(window_size);

getimage(160,100,430,250,window_buffer);

setviewport $(160,100,430,250,1)$;

m.menubox ();

out(10,25,"INTEL Microprocessors");

out(10,35,"MOTOROLA Microprocessors");

out(10,45,"IBM Microprocessors");

out(10,55,"SUNMAC Microprocessors");

setviewport $(0,0,639,469,1)$;

putimage(160,100,window_buffer,0);

free(window_buffer); 
An Acknowledgement Module is as follows,

void ackn(int aistsig,int asigstrt,int asigdest)

\{for(asigstrt;asigstrt $<=$ asigdest; asigstrt++)

$\{$ int $\operatorname{mcx}=0, \mathrm{mcy}=0$;

line(mcx+asigstrt,mcy+aistsig,mcx+asigstrt,mcy+aistsig);

line(mcx+asigdest, $m c y+$ aistsig, $m c x+$ asigdest-

6, mcy+aistsig-4);

line(mcx+asigdest, mcy+aistsig, $m c x+$ asigdest-

6 ,mcy+aistsig+4); \}\}

A Sample code for multi runs is as follows,

case 'd' : execvp("dma.exe",argv);

perror("exec error"); break;

case 'i' :execvp("i8259.exe",argv);

perror("exec error"); break;

case 'p' : execvp("ppi.exe",argv);

perror("exec error"); break;

Some of the characteristics followed in the present process

- Design should be user friendly.

- Minimum documentation is used and few templates are defined.

- More reusable components are used and minimized branching delay between components.

- An Interface should be designed so that it invokes the user's next requirement.

- The interface should communicate the status of any task introduced by the user.

- The use of navigation controls, menus, icons, and attributes such as colour, shape, layout etc.

- The interface should facilitate user involvement throughout the page and application design.

- The design of the any project applicationand its interface should improve the user's work efficiency but not the efficiency of the design engineer. It should improve the user's creativity.

- A vast library of reusable both hardware and user interface objects are developed.

\section{SIMULATION}

The present software support multiple simulations where can be process large data sets from the previous simulated results. Some of the results are presented in this section below. Here multiple simulations with different parameters are processed in single simulation model. The present simulation model view integrated with multiple executable file links. It provides flexibility in accessing multiple files in the same file. In a single window multiple runs can be made to meet the required design.

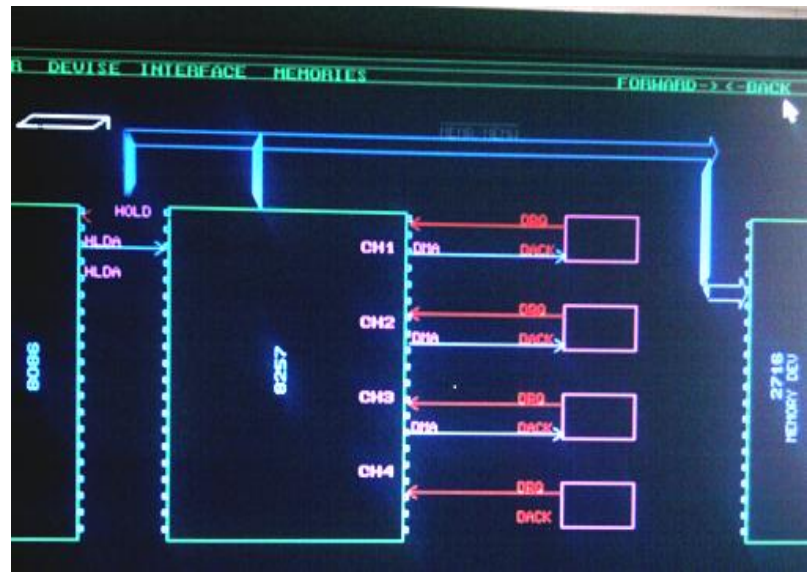

Figure 1: Basic principle operation of Direct Memory Accees

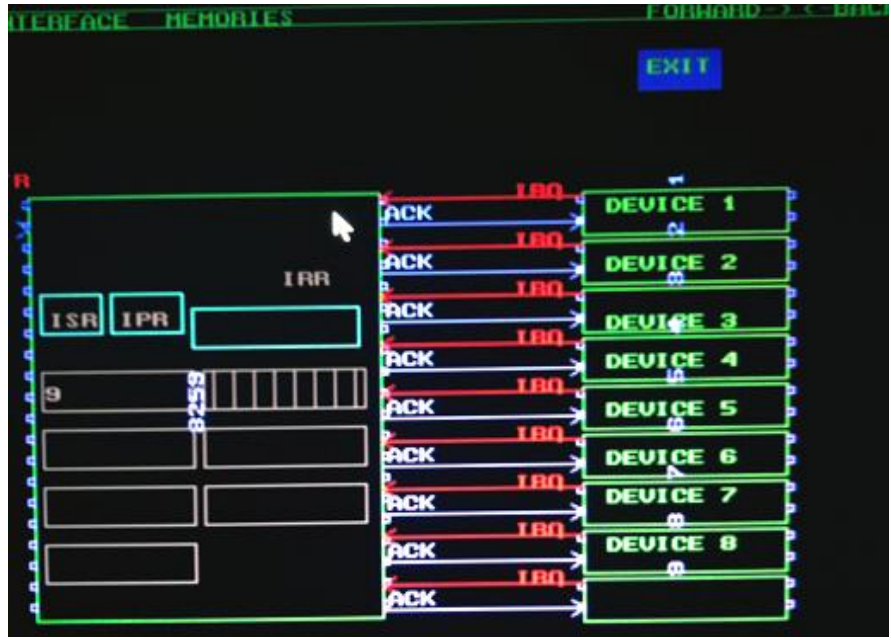

Figure 2: Basic principle operation of Interrupt Controller

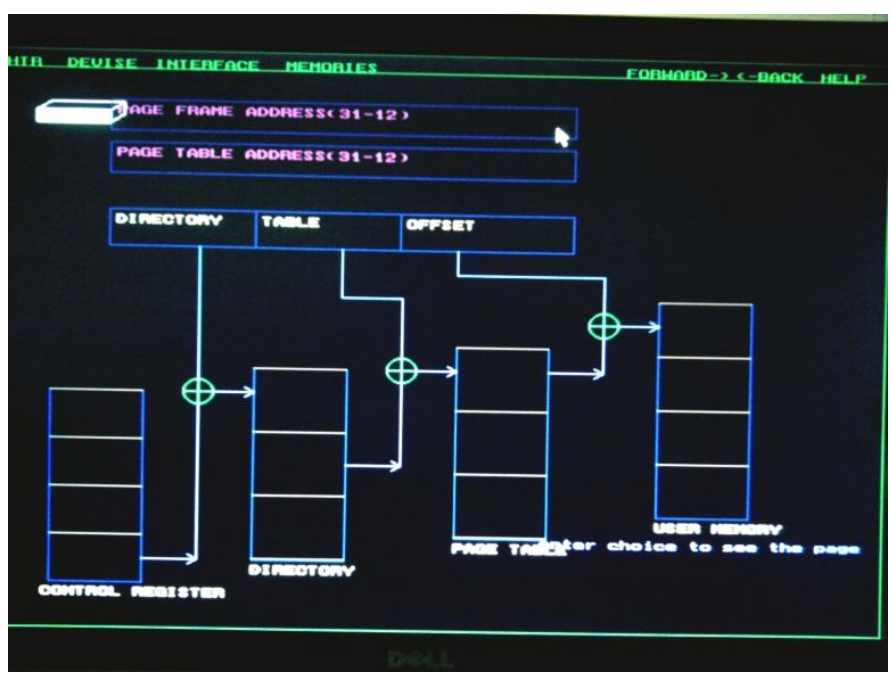

Figure 3: Memory Paging technique 


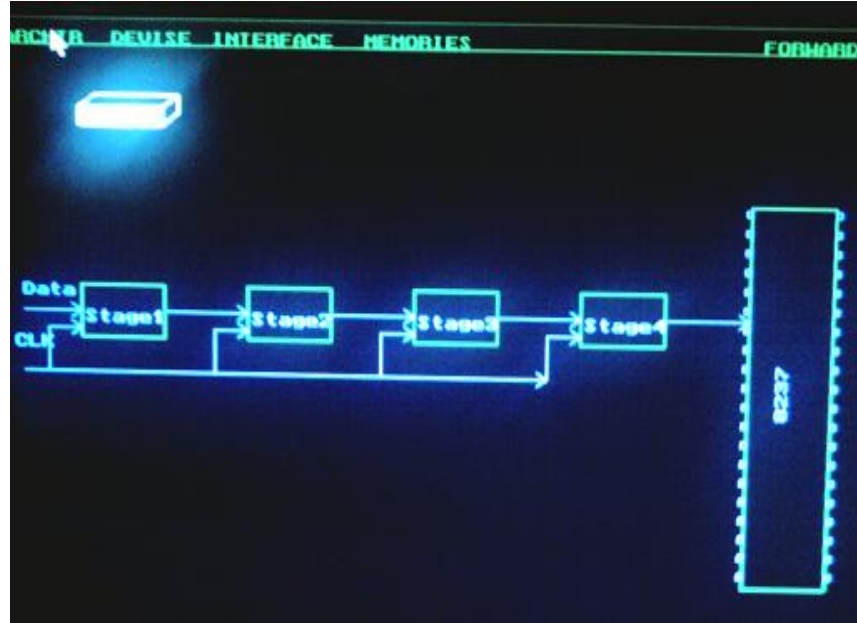

Figure 4: Analysing a research work: DMA with pipeline

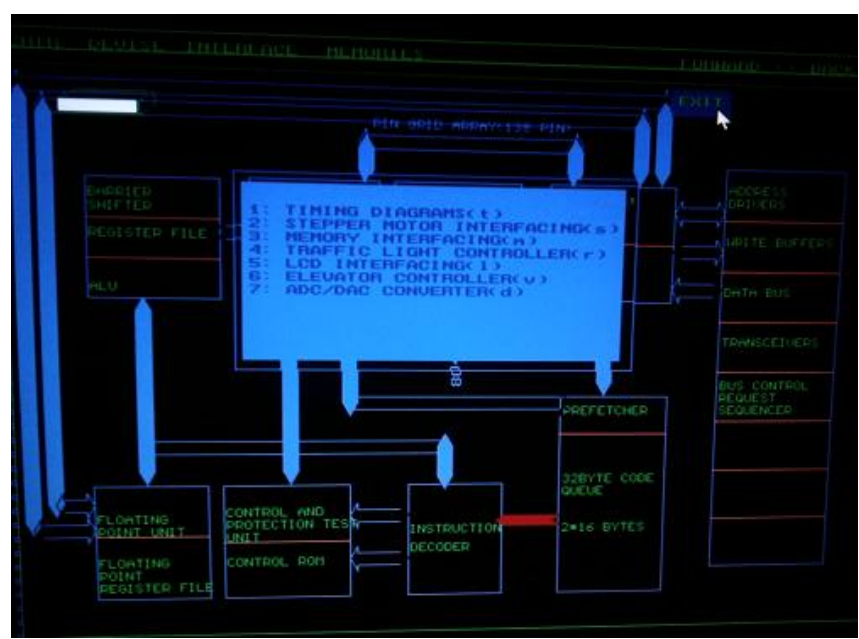

Figure 5: Architecture of $\mathbf{8 0 1 8 4}$ Microprocessor

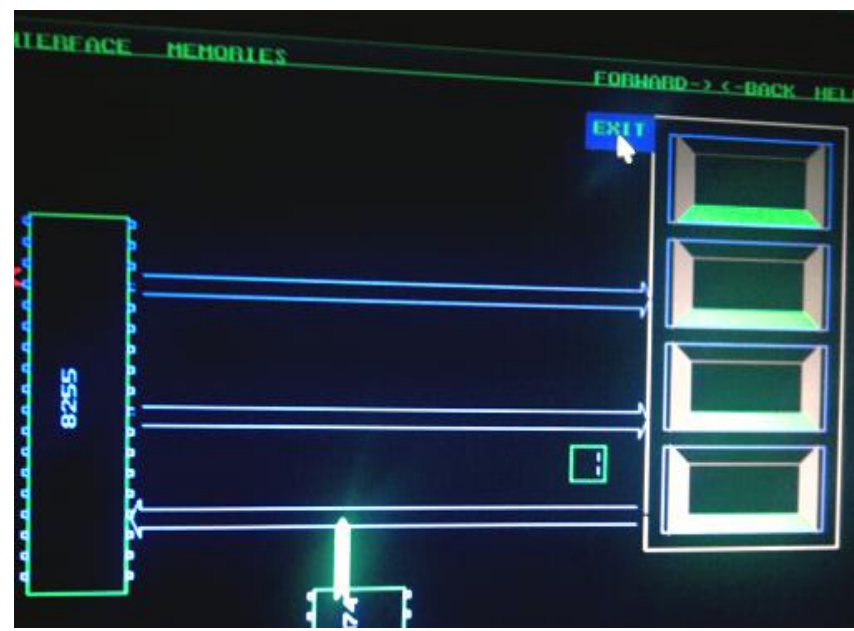

Figure 6: Elevator interfacing

In figure 1and Figure 2 the functional operations of DMA controller and Programming Interrupt Controller [1]can be analysed as, how the data bus is shifting from main processor 8086 to DMA controller. Simultaneously if anybody interested to know about 8086 microprocessor, the internal architecture module can be simulated with an exe file link. This is done simply by clicking on microprocessor block in the diagram. For instance consider some simulations. In figure 3 memory page techniques are simulated. In figure 4 a pipeline circuit from a research project [6] design is simulated andstudied the behaviour of pipeline with DMA. In figure 5 it is showing how multiple links are executed from one module. The links will be displayed on a small window like applet and can be selected by selected their specific code with numbers or characters. And then the corresponding exe file will be linked with present module. An application of multiple run is shown in figure 6 . In figure 6 the four main files are internally linked and simultaneously running. The microprocessor, Programmable Peripheral Interface (8255), Seven Segment Display and Elevator controller modules are executed in one single module by accessing their corresponding data from different text files which are created and updated in the program.

\section{TESTING}

Organizations need to face many challenges in triggering sustainable Information Communication Technology (SICT). SICT Capability Maturity frame work (ICT-CMF) assessment methodology determines overall sustainability goals and objects [9]. In the present work we set some series of questions to meet the goals and objects for ICT to meet economical, legacy sustainability.

- Does the organization recognize the ICT as major contributor?

- Is the ICT meeting all the goals and objectives of the end user?

- How frequently the designer reviewing the feedback from the end users?

- How rapidly the user requirements are changing as per the new technologies and their flexibility in operating the software?

- Are the user requirements are reliable?

- Set some new alterations in the component design after negotiations with the end user.

The testing aims to find faults, software's reliability, reusability, error prone and many other issues. If a single test finds a fault, it has certainly proven useful for the first purpose. However, when a single test does not uncover all faults, its usefulness in establishing a reliability estimate is severely limited. Statistical analysis of testing is designed for test suites, but some of this analysis is also applicable to a single test. Single test does not yield much useful information unless it uncovers a fault [5].In the present paper testing procedure is done effectively through following steps.

The testing includes following steps at class room environment.

\section{Stage 1:}

- Develop assignments

- Conduct alpha and beta testing

- $\quad$ Seek the student feed back

- Propose minor alterations in the current modules Stage 2:

- Ask the student to create his ownassignments

- Conduct quiz and descriptive test

- Propose alterations, deletion, insertion and development in the software if any based on stage 1 and stage 2 feedbacks.

- By analysing and doing frequent assessment, comparatively these stages manage sustainable ICT than the methods prosed in the previous studies [4]. 


\section{FEATURES}

- It is appropriate for the investigation and simulation purpose.

- The user's expected content and essential functions that are relevant to the user's needs are present in the software modules.

- It is designed traditionally, user friendly and visual appeal such as content, interface layout, colour coordination, the balance of text, graphics and other media, navigation mechanisms must attractive to end-users.

- It is Compatible withappropriate environments and configurations

- It reduces wear and tear and minimizes the project budget.

- More Environment friendly compared to Hardware.

- Helps Researchers to take immediate decisions.

- The software structure is very interactive and attractive.

- Multiple Simulations can be done.

- By further development of the modules in the software, it can use for research and real time application problems.

\section{CONCLUSION}

The present software is integrated with multiple links to interface internal blocks of a system. Modules are individually developed to allow reusability and development in the product. The software is extensively used for education and also helpful for researchers to analyse and study the behaviour of the digital systems. The simulation is very user friendly and easy to learn and use the predefined functions.

\section{REFERENCES}

[1] Kumar.N et al., "Effect of Interrupt Logic on Delay Balancing Circuit", International Journal of Computer Applications, Vol 27- No.4, August 2011.
[2] Robert S. Friedman and Fadi P. Deek, "Innovation and Education in the Digital Age: Reconciling the Roles of Pedagogy, Technology, and the Business of Learning" IEEE Transactions on engineering management, VOL. 50, NO. 4, Nov 2003,pg 403.

[3] Vasyl Zayats, Vasyl Kogut, "Role of Information Technologies in Progress of Science and Education" MEMSTECH'2009, 22- 24 April, 2009, PolyanaSvalyava (Zakarpattya), UKRAINE.

[4] Kumar, N.S., "Modern Computer Graphics Technologies Used at Educational Programs and Some Graphical output screens", (IJCSIS) International Journal of Computer Science and Information Security, Vol. 8, No. 3, June 2010.

[5] Keith Miller et al., "Software Test Cases: Is One Ever Enough?", IT Pro January I February 2006 Publish ed by t h e IEEE Computer Society, pp 44-48.

[6] Kumar.S et al., "A New Method to Enhance Performance of Digital Frequency Measurement and Minimize the Clock Skew", IEEE Sensors journal, vol. 11, no. 10, october 2011, pp.2421-2425.

[7] Xiping Song et al., "Engineering Software Design Processes to Guide Process Execution", IEEE Transactions on software engineering, vol. 24, no. 9, september 1998, pp. 759-775.

[8] Victoria L. Tinio, Webinars on "ICT in Education", This is set of E-Primers on the application of Information and Communication Technologies (ICTs) to development, is presented by UNDP for the benefit of participants to the World Summit on the Information Society.

[9] Donnellan, et al., " A Capability Maturity Framework for sustainable information and communication technology", Published by $\mathrm{t} h \mathrm{e}$ IEEE Computer Society, IT Pro January/February 2011, pg 33-40. 